\title{
THE INDEPENDENCE NUMBER OF THE ORTHOGONALITY GRAPH IN DIMENSION $2^{k}$
}

\author{
FERDINAND IHRINGER AND HAJIME TANAKA
}

\begin{abstract}
We determine the independence number of the orthogonality graph on $2^{k}$-dimensional hypercubes. This answers a question by Galliard from 2001 which is motivated by a problem in quantum information theory. Our method is a modification of a rank argument due to Frankl who showed the analogous result for $4 p^{k}$-dimensional hypercubes, where $p$ is an odd prime.
\end{abstract}

\section{INTRODUCTION}

The orthogonality graph $\Omega_{n}$ has the elements of $\{-1,1\}^{n}$ as vertices, and two vertices are adjacent if they are orthogonal, in other words, if their Hamming distance is $n / 2$. The graph $\Omega_{n}$ occurs naturally when comparing classical and quantum communication [3. In particular, for $n=2^{k}$ the cost of simulating a specific quantum entanglement on $k$ qubits can be reduced to determining the chromatic number $\chi\left(\Omega_{n}\right)$ of $\Omega_{n}$ [2, 9]. The graph $\Omega_{n}$ is edgeless if $n$ is odd, and is bipartite if $n \equiv 2(\bmod 4)$. For $n \equiv 0(\bmod 4)$, Frankl [7] and Galliard 9] constructed an independent set of $\Omega_{n}$ of size

$$
a_{n}:=4 \sum_{i=0}^{n / 4-1}\left(\begin{array}{c}
n-1 \\
i
\end{array}\right),
$$

and Galliard [9] asked in 2001 if this is the independence number $\alpha\left(\Omega_{n}\right)$ of $\Omega_{n}$ when $n=2^{k}, k \geqslant 2$. Newman [15] and, according to [8, p. 275, Remark], Frankl conjectured that this holds whenever $n \equiv 0(\bmod 4)$. See also [4. Frankl [7] already showed the conjecture in 1986 for all $n=4 p^{k}$ for $k \geqslant 1$, where $p$ is an odd prime. De Klerk and Pasechnik 13 proved the conjecture for $n=16$, i.e., that $\alpha\left(\Omega_{16}\right)=2304$, using Schrijver's semidefinite programming bound [16. Furthermore, Frankl and Rödl 8 showed that $\alpha\left(\Omega_{n}\right)<1.99^{n}$ if $n \equiv 0(\bmod 4)$. In this note, we apply Frankl's method from [7] to show the following:

Theorem. Let $n=2^{k}$ for some $k \geqslant 2$. Then $\alpha\left(\Omega_{n}\right)=a_{n}$.

Together with the discussion in [9, Section 5.5], that is using $\chi\left(\Omega_{n}\right) \geqslant 2^{n} / \alpha\left(\Omega_{n}\right)$, our result implies an explicit version of Theorem 4 in [2]. Finding such an explicit result is one motivation for Galliard's work. See also [10, 12.

The first author is supported by a postdoctoral fellowship of the Research Foundation Flanders (FWO).

The second author is supported by JSPS KAKENHI Grant Number JP17K05156. 


\section{Proof of the Theorem}

Let $A_{j}$ be the 0-1-matrix indexed by the vertices of the hypercube $Q_{n}=\{-1,1\}^{n}$ with $\left(A_{j}\right)_{x y}=1$ if $x$ and $y$ have Hamming distance $j$. The matrices $A_{j}$ have $n+1$ common eigenspaces $V_{0}, V_{1}, \ldots, V_{n}$, and in the usual ordering of the eigenspaces the eigenvalue of $A_{j}$ with respect to $V_{i}$ is given by the Krawtchouk polynomial (see [5, Theorem 4.2])

$$
K_{j}(i)=K_{j}(i ; n):=\sum_{h=0}^{j}(-1)^{h}\left(\begin{array}{l}
i \\
h
\end{array}\right)\left(\begin{array}{l}
n-i \\
j-h
\end{array}\right) .
$$

It is known that the orthogonal projection matrix $E_{i}$ onto $V_{i}$ has the entry $\left(E_{i}\right)_{x y}=$ $2^{-n} K_{i}(j)$ if $x$ and $y$ are at Hamming distance $j$ [5, Theorem 4.2], so that we have in particular rank $E_{i}=\operatorname{trace} E_{i}=K_{i}(0)=\left(\begin{array}{c}n \\ i\end{array}\right)$. The $(n+1)$-dimensional matrix algebra spanned by $A_{0}=I, A_{1}, \ldots, A_{n}$ is called the Bose-Mesner algebra of $Q_{n}$.

Assume now that $n=2^{k}, k \geqslant 3$. (The result is trivial if $k=2$.) Let $C$ be an independent set of $\Omega_{2^{k}}$, and let $C_{\text {even }}^{ \pm}, C_{\text {odd }}^{ \pm} \subseteq\{-1,1\}^{2^{k}-1}$ be as in [7]: $C_{\text {even }}^{+}$is given by taking all the even-weight elements of $C$ that end with +1 , followed by truncating at the last coordinate, and the other three are analogous. Let $C^{\prime}$ be one of these four families. Then the Hamming distances in $C^{\prime}$ are even and unequal to $2^{k-1}$, so they lie in the following set:

$$
\left\{2 s: s=0,1, \ldots, 2^{k-1}-1, s \neq 2^{k-2}\right\} .
$$

Below we work with the Bose-Mesner algebra $\mathscr{A}$ of $Q_{2^{k}-1}$. For every $M \in \mathscr{A}$, let $\bar{M}$ denote the principal submatrix corresponding to $C^{\prime}$. Consider the polynomial

$$
\varphi(\xi)=\left(\begin{array}{c}
\xi / 2-1 \\
2^{k-2}-1
\end{array}\right) \in \mathbb{R}[\xi],
$$

and expand it in terms of the Krawtchouk polynomials $K_{i}(\xi)=K_{i}\left(\xi ; 2^{k}-1\right)$ :

$$
\varphi(\xi)=\sum_{i=0}^{2^{k-2}-1} c_{i} K_{i}(\xi)
$$

Let

$$
X=\sum_{j=0}^{2^{k}-1} \varphi(j) A_{j} \in \mathscr{A} .
$$

On the one hand, observe that $\bar{X}$ has only integral entries in view of (1), and an easy application of Lucas' theorem (cf. [6]) shows moreover that $\bar{X} \equiv \bar{I}(\bmod 2)$. In particular, $\bar{X}$ is invertible. On the other hand, from (2) we have

$$
X=2^{2^{k}-1} \sum_{i=0}^{2^{k-2}-1} c_{i} E_{i}
$$

It follows that

$$
\left|C^{\prime}\right|=\operatorname{rank} \bar{X} \leqslant \operatorname{rank} X \leqslant \sum_{i=0}^{2^{k-2}-1} \operatorname{rank} E_{i}=\sum_{i=0}^{2^{k-2}-1}\left(\begin{array}{c}
2^{k}-1 \\
i
\end{array}\right) .
$$

As $|C|=\left|C_{\text {even }}^{+}\right|+\left|C_{\text {even }}^{-}\right|+\left|C_{\text {odd }}^{+}\right|+\left|C_{\text {odd }}^{-}\right|$, the theorem follows. 


\section{Future WORK}

Schrijver's semidefinite programming bound has been extended to hierarchies of upper bounds; see, e.g., [1, 14. In view of [13, it is interesting to investigate if these bounds in turn prove the conjecture for other values of $n$. One of the referees pointed out to us that using next level in the hierarchy, see [11, yields the correct bound of $a_{24}=178208$ for the case $n=24$.

Problem. Prove the conjecture for $n=40$, which is the first open case.

Acknowledgements. We thank the anonymous referee for solving the case $n=24$.

\section{REFERENCES}

[1] C. Bachoc, D. C. Gijswijt, A. Schrijver, and F. Vallentin, Invariant semidefinite programs, in: Handbook on semidefinite, conic and polynomial optimization (M. F. Anjos and J. B. Lasserre, eds.), Springer, New York, 2012, pp. 219-269; arXiv 1007.2905

[2] G. Brassard, R. Cleve, and A. Tapp, Cost of exactly simulating quantum entanglement with classical communication, Phys. Rev. Lett. 83 (1999) 1874-1877; arXiv quant-ph/9901035.

[3] H. Buhrman, R. Cleve, and A. Widgerson, Quantum vs. classical communication and computation, in: Proceedings of the 30th Annual ACM Symposium on the Theory of Computing, Dallas, TX, USA, 1998, pp. 63-68; arXiv quant-ph/9802040.

[4] P. J. Cameron, Problems from CGCS Luminy, May 2007, European J. Combin. 31 (2010) 644-648.

[5] P. Delsarte, An algebraic approach to the association schemes of coding theory, Philips Res. Rep. Suppl., No. 10, 1973.

[6] N. J. Fine, Binomial coefficients modulo a prime, Amer. Math. Monthly 54 (1947) 589-592.

[7] P. Frankl, Orthogonal vectors in the $n$-dimensional cube and codes with missing distances, Combinatorica 6 (1986) 279-285.

[8] P. Frankl and V. Rödl, Forbidden intersections, Trans. Amer. Math. Soc. 300 (1987) 259-286.

[9] V. Galliard, Classical pseudo-telepathy and colouring graphs, diploma thesis, ETH Zurich, 2001; available at http://math.galliard.ch/Cryptography/Papers/PseudoTelepathy/ SimulationofEntanglement.pdf .

[10] V. Galliard, A. Tapp, and S. Wolf, The impossibility of pseudo-telepathy without quantum entanglement, in: Proceedings 2003 IEEE International Symposium on Information Theory, Yokohama, Japan, 2003; arXiv quant-ph/0211011

[11] D. C. Gijswijt, H. D. Mittelmann, and A. Schrijver, Semidefinite code bounds based on quadruple distances, IEEE Trans. Inform. Theory 58 (2012) 2697-2705; arXiv 1005.4959

[12] C. D. Godsil and M. W. Newman, Coloring an orthogonality graph, SIAM J. Discrete Math. 22 (2008) 683-692; arXiv math/0509151

[13] E. de Klerk and D. V. Pasechnik, A note on the stability number of an orthogonality graph, European J. Combin. 28 (2007) 1971-1979; arXiv math/0505038

[14] M. Laurent, Strengthened semidefinite programming bounds for codes, Math. Program. 109 (2007) 239-261.

[15] M. W. Newman, Independent sets and eigenspaces, thesis, University of Waterloo, 2004.

[16] A. Schrijver, New code upper bounds from the Terwilliger algebra and semidefinite programming, IEEE Trans. Inform. Theory 51 (2005) 2859-2866.

Department of Mathematics: Analysis, Logic and Discrete Mathematics, Ghent UniVERSITY, BELGiUm

Email address: ferdinand.ihringer@ugent.be

Research Center for Pure and Applied Mathematics, Graduate School of InformaTiON SCIENCES, TOHOKU University, JAPAN

Email address: htanaka@tohoku.ac.jp 